$$
\begin{gathered}
\text { UCRL-JC-123003 } \\
\text { PREPRINT } \\
1196050369 \\
\text { CONF- } 960425--1 /
\end{gathered}
$$

\title{
Magnetic Force Microscopy of Single-Domain Cobalt Dots Patterned Using Interference Lithography
}

A. Fernandez, P. J. Bedrossian, S. L. Baker, S. P. Vernon, D. R. Kania

This paper was prepared for submittal to the International Magnetics Conference

Seattle, WA

April 9-12, 1996
RECEIVED

SEP O 9998

OSTI

March 20, 1996 


\section{DISCLAIMER}

This document was prepared as an account of work sponsored by an agency of the United States Government. Neither the United States Government nor the University of California nor any of their employees, makes any warranty, express or implied, or assumes any legal liability or responsibility for the accuracy, completeness, or usefulness of any information, apparatus, product, or process disclosed, or represents that its use would not infringe privately owned rights. Reference herein to any specific commercial product, process, or service by trade name, trademark, manufacturer, or otherwise, does not necessarily constitute or imply its endorsement, recommendation, or favoring by the United States Government or the University of California. The views and opinions of authors expressed herein do not necessarily state or reflect those of the United States Government or the University of California, and shall not be used for advertising or product endorsement purposes. 


\section{DISCLAIMER}

This report was prepared as an account of work sponsored by an agency of the United States Government. Neither the United States Government nor any agency thereof, nor any of their employees, makes any warranty, express or implied, or assumes any legal liability or responsibility for the accuracy, completeness, or usefulness of any information, apparatus, product, or process disclosed, or represents that its use would not infringe privately owned rights. Reference herein to any specific commercial product, process, or service by trade name, trademark, manufacturer, or otherwise does not necessarily constitute or imply its endorsement, recommendation, or favoring by the United States Government or any agency thereof. The views and opinions of authors expressed herein do not necessarily state or reflect those of the United States Government or any agency thereof. 


\section{DISCLAIMER}

Portions of this document may be illegible in electronic image products. Images are produced from the best available original document. 


\title{
Magnetic Force Microscopy of Single-Domain Cobalt Dots Patterned Using Interference Lithography
}

A. Fernandez, P. J. Bedrossian, S. L. Baker, S. P. Vernon, and D. R. Kania Lawrence Livermore National Laboratory, Livermore, CA 94550

\begin{abstract}
We have fabricated arrays of Co dots having diameters of $100 \mathrm{~nm}$ and $70 \mathrm{~nm}$ using interference lithography. The density of these arrays is $7.2 \times 10^{\%} / \mathrm{in}^{2}$. Magnetic force microscopy measurements indicate that the $C_{0}$ dots are single-domain with moments that can be controlled to point either in-plane or out-of-plane. Interference lithography is a process that is easily scaled to large areas and is potentially capable of high throughput. Large, uniform arrays of single-domain structures are potentially useful for high-density, low-noise data storage.
\end{abstract}

\section{INTRODUCTION}

It is anticipated that an alternate form of magnetic media will be required for very high-density data storage. The packing density of thin-film recording media is fundamentally limited by the jaggedness of domain walls which gives rise to noise in the readback signal [1]. In principle, a storage technology based on single magnetic domain particles can overcome this limitation and allow for much higher storage densities [2]-[4]. A single-domain particle can be magnetized in two discrete states with equal but opposite magnetic moments. An array of such particles could be used to record binary information with each particle storing one bit of information.

Recently, several groups have investigated the magnetic properties of small, lithographically defined magnetic structures and considered the possibility of their use for data storage [2]-[4]. New et al. fabricated arrays of $150 \mathrm{~nm}$ by $200 \mathrm{~nm}$ islands patterned from $20 \mathrm{~nm}$ thick Co films [2], [3]. They used electron beam lithography to define the islands and a dry etch process to transfer the pattern. Magnetic force microscopy (MFM) indicates that the islands are single-domain with moments that are in-plane [3]. Using electron beam lithography and electroplating, Krauss et al. fabricated arrays of $50 \mathrm{~nm}$ diameter $\mathrm{Ni}$ pillars with densities as high as $6.5 \times 10^{10} / \mathrm{in}^{2}$ [4]. These particles exhibit single-domain behavior with moments that are out-of-plane.

One of the main challenges in developing a storage technology based on single-domain particles is finding a

Manuscript received March 4, 1996.

A. Fernandez, e-mail Fernandez10@llnl.gov, phone 510-424-2904, FAX 510-422-8761. 
viable and cost-effective process for fabricating high-density arrays of magnetic structures. Three key fabrication requirements must be satisfied. (1) The particles must be sufficiently small to inhibit multiple domain formation. This requires that the particles be approximately $100 \mathrm{~nm}$ or less in diameter. (2) The size and shape of the particles must be uniform over large areas. (3) The arrays must be produced inexpensively and with a process that can be easily scaled to meet the demands of high-volume manufacturing.

In this paper, we report on the use of interference lithography to fabricate high-density arrays of single-domain Co dots. Interference lithography provides a relatively inexpensive way to generate periodic structures over large areas. It has been used in a variety of applications including the fabrication of $\mathrm{x}$-ray and extreme ultra-violet gratings [5], ultra-violet polarizers and filters [6], and, more recently, field emission arrays for flat panel displays [7]-[9]. Importantly, interference lithography is capable of defining structures with dimensions of $100 \mathrm{~nm}[5]-[10]$. Here, we present MFM analysis of the fabricated $\mathrm{Co}$ arrays. These measurements demonstrate we can control the dimensions of the structures to permit easy-axis magnetization either in-plane or out-of-plane.

\section{FABRICATION}

Interference lithography is carried out by combining two beans of coherent radiation at a specified angle at the exposure plane. Interference of the two beams produces a sinusoidal intensity pattern with a period given by $\lambda /[2 \sin (\theta)]$ where $\lambda$ is the wavelength and $\theta$ is the recombination half-angle. If the beam intensities are matched, the modulation transfer function of the intensity pattern is 1 and independent of the period. This has the important consequence that deep submicron period gratings can be produced with a near $i$-line coherent source. (We use the $351 \mathrm{~nm}$ line of an Ar ion laser.) Another advantage is that the depth-of-focus of the interference pattern is virtually infinite on the scale of semiconductor processing [10] due to the long coherence length of the lasers typically used. Furthermore, the exposure area can be scaled to areas as large as $50 \mathrm{~cm} \times 50 \mathrm{~cm}$ [9] which makes interference lithography attractive for high-throughput applications.

The details of the dot fabrication process are described elsewhere [9]. Briefly, a resist coated Si wafer is exposed to the interference pattern. It is subsequently exposed a second time after being rotated $90^{\circ}$ with respect to the first pattern. Development of the exposed layer produces a 2-dimensional array of resist holes or pedestals. Co dots are defined by thermally evaporating a thin layer of Co through the holes and subsequently using a lift-off process. No intermediate film is deposited between the $\mathrm{Co}$ and $\mathrm{Si}$. The dots are centered on a $300 \mathrm{~nm}$ square grid which 


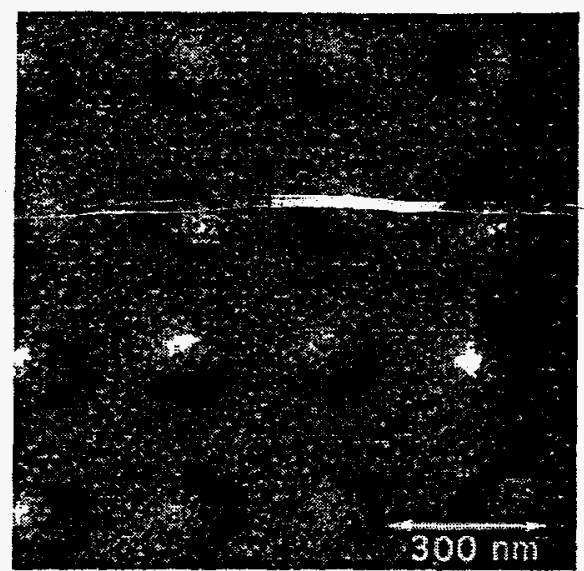

Fig. 1. MFM image of an array of type I Co dots which are $100 \mathrm{~nm}$ in diameter and $40 \mathrm{~nm}$ tall. The dipole signature of these dots indicates the moments are in-plane.

corresponds to a density of $7.2 \times 10^{9} / \mathrm{in}^{2}$. Their diameter is controlled by varying the exposure dose.

Two types of samples are fabricated for this study. In the first type (referred to as type I), the nominal dot diameter is $100 \mathrm{~nm}$ and the Co thickness is $40 \mathrm{~nm}$. In the second type (type II), the nominal dot diameter is reduced to $70 \mathrm{~nm}$ while the Co thickness is increased to $120 \mathrm{~nm}$. A characteristic of this process is that type-II dots are shaped like cones. Cones are formed because the diameter of the holes decrease during deposition due to condensation of $\mathrm{Co}$ on the periphery of the holes. Consequently, as the deposition continues, cone shaped dots grow at the bottom of the holes. The tips of the cones are formed as the apertures close. The average height of the type II dots is $100 \mathrm{~nm}$ as measured with an atomic force microscope (AFM). It follows from the above argument that type-I Co dots are shaped like truncated cones with a height of $40 \mathrm{~nm}$.

\section{MAGNETIC FORCE MICROSCOPY}

MFM measurements are performed on a Nanoscope Dimension 5000 AFM. All MFM images are phase images acquired using Digital Instruments' patented LiftMode [11] technique. Fig. 1 shows an MFM image of an array of type I Co dots. The characteristic dipole-like shape of the dots indicates that each dot is uniformly magnetized in a direction parallel to the substrate. This result is consistent with recent studies by New et al. [3] which show that patterned Co particles smaller than $200 \mathrm{~nm}$ are single-domain.

It is clear from Fig. 1 that the in-plane moments of these dots are not preferentially aligned. This is expected since they are circularly symmetric in the plane and therefore do not possess a strong azimuthal shape anisotropy. Dots with an elliptical shape can be fabricated with this process by varying the relative exposure dose for the two orthogonal interference exposures. 


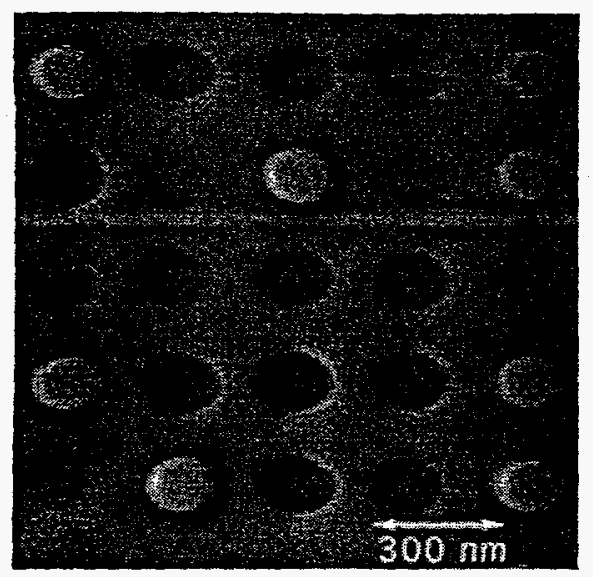

Fig. 2. MFM image of an array of type II Co dots which are $70 \mathrm{~nm}$ in diameter and $100 \mathrm{~nm}$ tall. The symmetry of the images shows that the moments are out-of-plane.

By varying the aspect ratio of the dots out of the plane, we are able to control the direction of magnetization. In Fig. 2 is an MFM image of type II Co dots which are taller and narrower than type I dots. The images of the dots are primarily azimuthally symmetric and either darkindicating a negative force gradient-or light-indicating a positive force gradient. We interpret these images to show that the Co dots are single-domain and magnetized in a direction which is normal to the plane of the substrate. We find that over $95 \%$ of the type II Co dots are magnetized in an out-of-plane direction.

Figs. 3(a) and 3(b) show MFM images of type II Co dots after application of a strong magnetic field along the surface normal in a direction parallel and anti-parallel to the tip moment respectively. From these images we learn that dark dots correspond to moments which are parallel to the tip moment while light dots correspond to dots which are anti-parallel. A slightly larger percentage of dots are magnetized parallel to the tip in Fig. 3(a) compared those that are anti-parallel in Fig. 3(b). This is attributed to a weak but discernible tendency for anti-parallel moments to flip during scanning to a parallel configuration. As an example, in the lower right comer of Fig. 3(b) are two dots which flipped as the MFM tip passed over them. (The image was scanned from top to bottom.) Fabricating dots with a stronger shape anisotropy should minimize this effect.

\section{CONCLUSIONS}

We have fabricated arrays of Co dots with diameters of $100 \mathrm{~nm}$ and smaller with an areal density of $7.2 \times 10^{9} / \mathrm{in}^{2}$ using laser interference lithography. MFM analysis of the dots indicates that they are single-domain with moments that can be controlled to point either in-plane or out-of-plane. While a fabrication process based on lift-off has been used here, it should be possible to couple other fabrication schemes such as dry etching and electroplating 

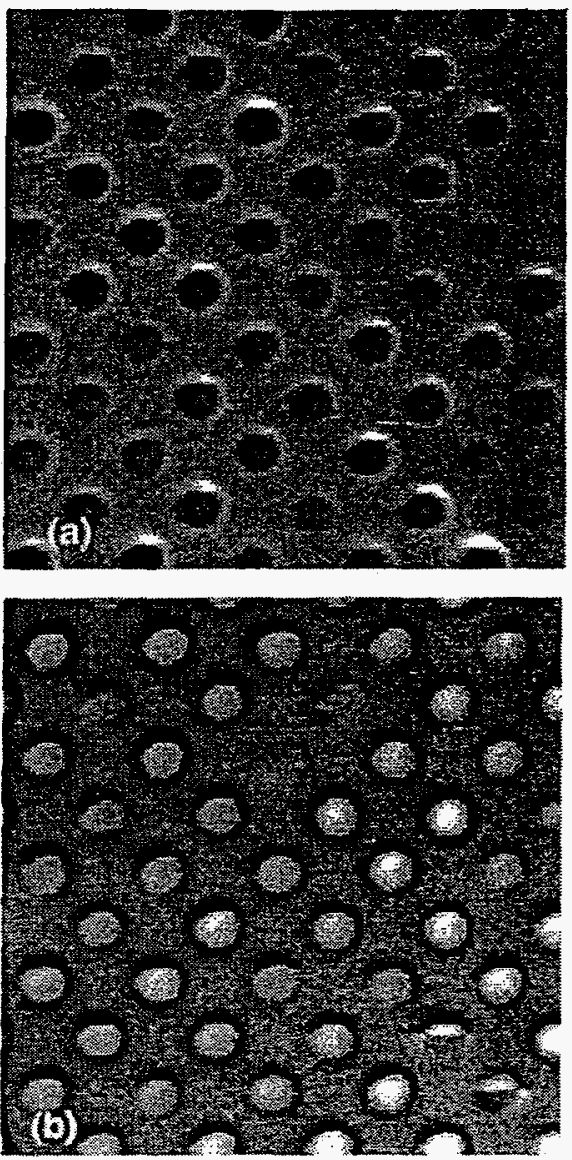

Fig. 4. MFM images of an array of type II Co dots after application of a strong magnetic field along the surface normal in a direction (a) parallel and (b) anti-parallel to the MFM tip moment.

with interference lithography to pattern arrays of small magnetic structures with a wide variety of 3-dimensional shapes. The distinct advantages of using interference lithography over other lithographic techniques are that high-density arrays of nanometer scale structures can be produced over large areas, in a relatively inexpensive way, and with potentially large throughput.

\section{ACKNOWLEDGMENT}

This work was performed under the auspices of the U. S. Department of Energy by the Lawrence Livermore National Laboratory under contract no. W-7405-Eng-48.

\section{REFERENCES}

[1] A. M. Barany and H. N. Bertram, "Transition noise model for longitudinal thin-film media," IEEE Trans. Mag., vol. 23, pp. 1776-1788, March 1987.

[2] R. M. H. New, R. F. W. Pease, and R. L. White, "Physical and magnetic properties of submicron lithographically patterned islands", J. Vac. Sci. Technol. B, vol. 13, pp. 1089-1094, May/June 1995.

[3] R. M. H. New, R. F. W. Pease, and R. L. White, "Effect of magnetocrystalline anisotropy in single-domain polycrystalline cobalt islands," IEEE Trans. Mag., vol. 31, pp. 3805-3807, Nov 1995. 
[4] P. R. Krauts and S. Y. Chou, "Fabrication of planar quantum magnetic disk structure using electron beam lithography, reactive ion etching, and chemical mechanical polishing," J. Vac. Sci. Technol. B, vol. 13, pp. 2850-2852, Nov/Dec 1995.

[5] M. L. Schattenburg, R.J. Aucoin, R.C. Fleming, I. Plotnick, J. Porter, and H. 1. Smith, "Fabrication of high energy $x$-ray transmission gratings for AXAF," SPIE Proc., vol. 2280, pp. 181-190, May 1994.

[6] E.E. Scime, E. H. Anderson, D. J. Mc Comas, and M. L. Schattenburg, "Extreme-ultraviolet polarization and filtering with gold transmission gratings," Applied Optics, vol. 34, pp. 648-654, Feb 1995.

[7] C.O. Bozler, C.T. Harris, S. Rate, D.R. Rathman, M. Hollis, and H.I. Smith, "Arrays of gated field-emitter cones having $0.32 \mu \mathrm{m}$ tip-to-tip spacing," J. Vac. Sci. Technol. B, vol. 12, pp. 629-632, Mar/Apr 1994.

[8] J. P. Spallas, A. M. Hawryluk, and D. R. Kania, "Field emitter array mask pattering using laser interference lithography," $J$. Vac. Sci. Technol. B, vol. 13, pp. 1973-1978, Sep/Oct 1995.

[9] J. P. Salas, R. D. Boyd, J. A Britten, A. Fernandez, A. M. Hawryluk, M. D. Perry, and D. R Kana, "Large scale field emitter array patterning for flat panel displays using interference lithography," J. Vac. Sci. Technol. B., in press.

[10] S. H. Zaidi and S. R. J. Brueck, "Multiple-exposure interference lithorgaphy," J. Vac. Sci. Technol. B, vol. 11, pp. 658-666, May/June 1993.

[11] LiftMode is a trademark of Digital Instruments. LiftMode, V. Flings and J. Gurley, U. S. Patent No. 5,308,974, Digital Instruments, Santa Barbara, Ca. 